Mehr Therapieauswahl bei CML und $\mathrm{Ph}+\mathrm{ALL}$

\title{
Dasatinib: Effektive Alternative bei Imatinib-Resistenz und -Intoleranz
}

In der Primärtherapie der chronischen myeloischen Leukämie (CML) gilt der BCR-ABLTyrosinkinase-Inhibitor Imatinib heute als Standard, da er die Progression der Erkrankung hemmt und das Überleben verlängert. Doch obwohl diese molekular zielgerichtete Substanz als Durchbruch in der CML-Therapie zu werten ist, gibt es weiterhin Probleme, z.B. aufgrund der Resistenzentwicklung. Hier steht jetzt mit dem Multi-Tyrosinkinase-Inhibitor Dasatinib ein neues «small molecule» zur Verfügung, das fast alle dieser Resistenzen überwinden kann und betroffenen Patienten damit eine neue effektive Therapieoption bietet. Dasatinib ist ein gezielt entwickeltes Designer-Molekül, das eine über 300fach höhere Wirkpotenz als Imatinib besitzt und sich auch bei Imatinib-Intoleranz bewährt hat.

Im Jahr 1990 - 145 Jahre nach der Erstbeschreibung der CML durch Virchow - wurde die BCR-ABL-Kinase, ein durch reziproke Translokation zwischen den Chromosomen 9 und 22 entstehendes Fusionsprotein, als pathogenetisch für diese Leukämie identifiziert. «Bei BCR-ABL handelt es sich um eine konstitutiv aktivierte Tyrosinkinase, die auf mehrere Signalwege wirkt und wichtige Zellfunktionen wie Proliferation, Apoptose und Adhäsion beeinflusst», erläuterte Nikolas von Bubnoff, München. Damit ist es eine ideale therapeutische Zielstruktur für die Behandlung Philadelphia-Chromosom-positiver $(\mathrm{Ph}+)$ Leukämien, die das Fusionsgen exprimieren. Seit Einführung des BCR-ABL-Kinase-Inhibitors Imatinib konnte das mediane Überleben von CML-Patienten von zuvor 3,5-5 Jahren auf hochgerechnet mehr als 15 Jahre verlängert werden. «Seit wir die CML in chronischer Phase intensiv behandeln können und so eine deutliche Reduktion der Tumorlast erreichen, hoffen wir, eine Blastenkrise meist völlig vermeiden zu können», konstatierte Andreas Hochhaus, Mannheim.

\section{Mehrjährige Therapie induziert Resistenzen}

Allerdings muss Imatinib dauerhaft gegeben werden, und das kann sich als problematisch erweisen. So entwickeln $60 \%$ bzw. über $90 \%$ der Patienten in der akzelerierten Phase bzw. in der Blastenkrise innerhalb von 3 Jahren unter der Therapie eine sekundäre Resistenz. In der chronischen Phase ist eine Resistenzentwicklung laut Hochhaus zwar seltener. Dennoch sind in früher chronischer Phase $5 \%$, in später chronischer Phase $13 \%$ der behandelten Patienten nach 5 Jahren resistent gegen den Tyrosinkinase-Inhibitor. Die Hälfte dieser Resistenzen ist durch Mutationen der ATP-bindenden Tyrosinkinase-Domäne (z.B. P-Loop-Mutationen) bedingt. «Bislang sind über 40 Mutationen des BCR-ABLGens bekannt. Sie sind sehr heterogen und induzieren unterschiedlich stark ausgeprägte Resistenzen. Insbesondere bei Mutationen, die zu einem steilen Anstieg der mittleren Hemmkonzentration führen, ist es wichtig, Imatinib rasch abzusetzen, um die klonale Selektion resistenter Zellen zu verhindern», erläuterte Hochhaus.

\section{Dasatinib: Höhere Wirkpotenz durch Multi-Targeting}

Mit Dasatinib (Sprycel ${ }^{\mathrm{TM}}$ ) steht jetzt ein alternativer BCR-ABL-Kinase-Inhibitor zur Verfügung, der wie Imatinib auch c-kit- und PDGFR-Kinasen blockiert und darüber hinaus auch die SRC-Kinasen hemmt. «Die neue Substanz bindet anders an die BCR-
ABL-Domäne als Imatinib, ist deshalb auch in vitro deutlich potenter», erklärte von Bubnoff. Ein weiterer Vorteil: Dasatinib ist strukturell nicht verwandt mit Imatinib und besitzt daher ein wenig überlappendes $\mathrm{Ne}$ benwirkungsspektrum. Präklinischen Untersuchungen zufolge werden alle bislang untersuchten BCR-ABL-Mutationen - mit Ausnahme der Mutation T315I - durch Dasatinib bereits in sehr niedriger Konzentration gehemmt. Zudem zeigt Dasatinib bei BCR-ABL-unabhängigen Resistenzen eine gute Wirksamkeit, was Hochhaus mit der Inhibition der SRC-Kinasen erklärte.

Der neue Kinase-Inhibitor wurde mittlerweile erfolgreich in allen Phasen der CML und bei der $\mathrm{Ph}+$ akuten lymphatischen Leukämie $(\mathrm{Ph}+\mathrm{ALL})$ bei Patienten geprüft, die gegen Imatinib resistent waren oder es nicht vertrugen. In der START-Studie sprachen $90 \%$ der CML-Patienten in chronischer Phase innerhalb von 6-12 Monaten mit einer kompletten hämatologischen Remission (CHR) auf Dasatinib $(2 \times 70 \mathrm{mg} / \mathrm{d})$ an. Die Rate zytogenetischer Remissionen (CyR) betrug $51 \%$, die kompletter zytogenetischer Remissionen (CCyR) 40\% (Abb. 1). Mit einer CyR-Rate von knapp $80 \%$ waren die Ergebnisse in der Subgruppe Imatinib-intoleranter Patienten besonders gut. «Doch auch bei Imatinib-Resistenz sprachen noch $42 \%$ der Patienten mit einer CyR an», betonte Hochhaus.

In späteren CML-Phasen (Studien START-A und -B) werden unter Dasatinib ebenfalls hohe hämatologische und zytogenetische Remissionsraten erreicht (Tab. 1). [Die vier START-Studien (-A, -B, -C, -L) umfassen einarmige offen Phase-II-Studien mit Patienten in allen Phasen der CML sowie Patienten mit Ph+ ALL.] «Die bisherigen Studiendaten belegen, dass ein Großteil der Patienten durch die Zweitlinientherapie mit Dasa-
Abb. 1. Effektivität von Dasatinib nach 6- bis 12-monatiger Therapie bei CML-Patienten in chronischer Phase $\mathrm{CHR}=$ komplette hämatologische Remission; $\mathrm{PCyR}=$ partielle zytogenetische Remission; $\mathrm{CCyR}=$ komplette zytogenetische Remission.

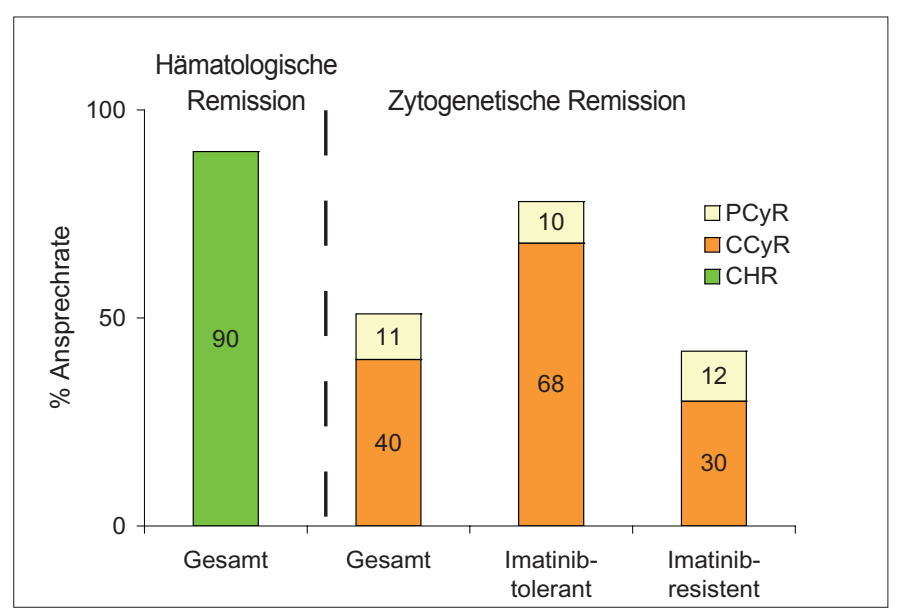




\section{Neu: Sprycel ${ }^{\mathrm{TM}}$ (Dasatinib) in Europa} zugelassen

München, 20. November 2006 - Der Wirkstoff Dasatinib, Handelsname Sprycel ${ }^{\mathrm{TM}}$, des Unternehmens Bristol-Myers Squibb ist seit heute durch die Europäische Kommission zur Behandlung der chronischen myeloischen Leukämie (CML) und Ph+ ALL zugelassen.

Sprycel $^{\mathrm{TM}}$ kann bei Erwachsenen eingesetzt werden, die sich in der chronischen Phase, der akzelerierten Phase oder in der Blastenkrise der Krankheit befinden und die therapieresistent sind oder intolerant auf bisherige Therapien einschließlich Imatinibmesilat reagieren.
Abb. 2. Randomisierte Vergleichsstudie mit hoch dosiertem Imatinib versus Dasatinib.

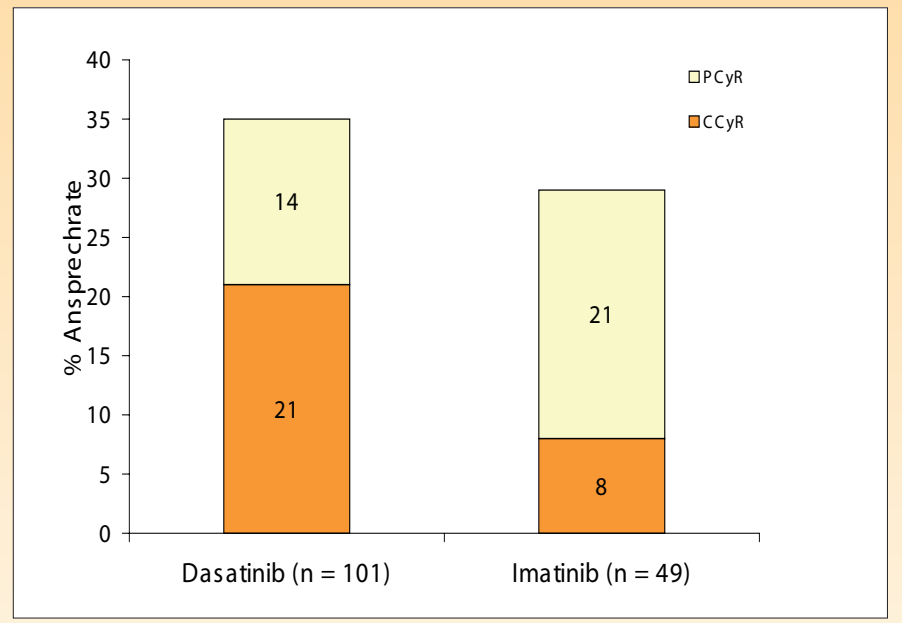

Tab. 1. Effektivität von Dasatinib in Phase-II-Studider CML und der $\mathrm{PH}+$

ALL. CP = chronische

Phase; $\mathrm{AP}=$ akzelerierte

Phase; MyBK = Myeloblas-

tenkrise; LyBK = lympha-

tische Blastenkrise; $\mathrm{NR}=$

nicht beschrieben. en in verschiedenen Phasen

\begin{tabular}{llllll}
\hline \multicolumn{5}{c}{ Patienten, $\mathrm{n}$} \\
\cline { 2 - 6 } & $\begin{array}{l}\text { CP } \\
(\mathrm{n}=387)\end{array}$ & $\begin{array}{l}\text { AP } \\
(\mathrm{n}=174)\end{array}$ & $\begin{array}{l}\text { MyBK } \\
(\mathrm{n}=109)\end{array}$ & $\begin{array}{l}\text { LyBK } \\
(\mathrm{n}=48)\end{array}$ & $\begin{array}{l}\text { Ph+ ALL } \\
(\mathrm{n}=46)\end{array}$ \\
\hline Major HR & NR & 59 & NR & 33 & 39 \\
CHR & 90 & 84 & 24,8 & 29 & 33 \\
Major CyR & 51 & 34 & 31 & 44 & 46 \\
$\begin{array}{l}\text { Komplette } \\
\text { CyR }\end{array}$ & 40 & 25 & 25 & 38 & 44 \\
\hline
\end{tabular}

tinib wieder in eine gute Remission gebracht werden kann», kommentierte Hochhaus.

\section{Therapieumstellung auf Dasatinib vorteilhaft}

In einer randomisierten Phase-II-Studie erfolgte darüber hinaus bei Imatinib-resistenten CML-Patienten in chronischer Phase ein Vergleich zwischen Dasatinib $(2 \times 70 \mathrm{mg} / \mathrm{d})$ und der Hochdosis-Therapie mit Imatinib $(800 \mathrm{mg} / \mathrm{d})$. Hier zeigt sich bereits nach kurzem Follow-up von nur 12 Wochen ein deutlicher Vorteil zugunsten der Therapieumstellung: So sprachen im Imatinib-Arm nur 29\%, im Dasatinib-Arm dagegen 35\% der Patienten mit einer CyR an (Abb. 2). Besonders ausgeprägt war die Überlegenheit von Dasatinib bei den CCyR (21 vs. $8 \%$ ).

Bei CML-Patienten in chronischer Phase mit suboptimalem Ansprechen auf Imatinib (z.B. keine CHR nach 3, keine CCyR nach 12 Monaten) werden derzeit mehrere Optionen wie eine Erhöhung der Imatinib-Dosis auf $800 \mathrm{mg}$, die Umstellung auf andere Kinase-Inhibitoren oder Kombinationen von
Imatinib und weiteren Medikamenten mit

\section{Erfolge bei $\mathrm{Ph}+\mathrm{ALL}$}

Auch bei Patienten mit Ph+ ALL führt Dasatinib in etwa $40 \%$ der Fälle zu einer guten hämatologischen Remission - unabhängig davon, ob eine Imatinib-Resistenz oder -Intoleranz vorliegt oder zuvor bereits eine Stammzelltransplantation vorgenommen wurde. Die Zulassung des neuen BCR-ABLKinase-Inhibitors für die Therapie der rezidivierten und refraktären $\mathrm{Ph}+\mathrm{ALL}$ ist deshalb nach Meinung von Oliver G. Ottmann, Frankfurt/M., als wichtiger Zugewinn zu werten, könnte sie doch auch rezidivierten Patienten, die in Remission kommen, noch eine Transplantation ermöglichen. Angelaufen sind jetzt weitere Studien, die Dasatinib in Kombination mit einer Chemotherapie prüfen. Als attraktives Konzept wertete Ottmann außerdem Erhaltungstherapien mit Dasatinib bei transplantierten Patienten.

Häufigste nichthämatologische Nebenwirkungen von Dasatinib sind Diarrhöen, Kopfschmerzen, oberflächliche Ödeme, Hautausdifferentem Wirkansatz geprüft. schläge und Pleuraergüsse, die jedoch überwiegend leicht (Grad 1/2) ausgeprägt sind. In schweren Fällen plädierte Alois Gratwohl, Basel, für ein vorübergehendes Absetzen der Substanz und eine Reexposition nach Abklingen der Beschwerden. Zusätzlich kann eine symptomatische Therapie mit Diuretika, Loperamid und Analgetika oder eine Dosisreduktion hilfreich sein. «Bei respondierenden Patienten, die unter Dasatinib eine Zytopenie entwickeln, sollte die Therapie unbedingt weitergeführt und zusätzlich supportiv und mit hämatopoetischen Wachstumsfaktoren behandelt werden», empfahl Gratwohl.

\section{Quelle}

Satellitensymposium «Imatinib - Resistenz: Quo vadis?» und Pressekonferenz «CML. State of the Art» anlässlich der Gemeinsamen Jahrestagung der Deutschen, Österreichischen und Schweizerischen Gesellschaften für Hämatologie und Onkologie, Leipzig, 5. November 2006.

\section{Bericht}

Dr. Katharina Arnheim, Berlin

\section{Impressum}

Mehr Therapieauswahl bei CML und Ph+ ALL Dasatinib: Effektive Alternative bei Imatinib-Resistenz und -Intoleranz

PharmaForum in ONKologie 30 | 1 | 07

C 2007 by S. Karger Verlag für Medizin und Naturwissenschaften $\mathrm{GmbH}$ Lörracher Straße 16a 79115 Freiburg, Deutschland

Mit freundlicher Unterstützung von Bristol-Myers Squibb GmbH \& Co. KGaA.

Der Verlag und die Herausgeber der Zeitschrift übernehmen keine Verantwortung für diese Rubrik.

\section{KARGER ○ 2007 S. Karger GmbH, Freibur}

